\title{
Phonemic recoding of figural information and memory span
}

\author{
STEFAN SLAK, KATHLEEN M. KELLEY, and JONELLE SKIBSKI \\ University of Toledo, Toledo, Ohio 43606
}

\begin{abstract}
The purpose of the present study was to verify the effect of phonemic recoding of figural items on memory span in two individual subjects who, prior to the experiment proper, learned a phonemic code allowing them to recode bivariate figural items into pronounceable twophoneme syllables. Memory span was determined with the use of a phonemic code and with the use of the natural language where subjects in a memory span task read the items aloud in terms of the phonemic code or the natural language, respectively, and reproduced the items in figural form in both cases. The hypothesis that reduction in the length of verbal representation with the use of phonemic recoding would increase the memory span was supported by the results. Phonemic recoding was compared with the traditional phonetic mnemonic system.
\end{abstract}

Slak (1970) demonstrated that phonemic recoding of digital information, whereby triplets of decimal digits are recoded into pronounceable three-phoneme syllables and at the time of recall decoded back into the digital form, significantly increases not only shortterm memory capacity, but also the rate of serial learning, free recall, and memory recognition. The effect was explained in terms of reduction in the amount of verbal representation that stands for a given amount of information, the amount measurable in number of phonemes. "Reducing representational redundancy for a given source of information results in a greater storability of that information, but the relative increment in storability is less than relative decrement in representational redundancy" (Slak, 1970, p. 406). The benefit of recoding is less than ideal, nor is the increase in processing efficiency cheap for the subject who has to undergo many hours of code learning before he acquires the skill of being able to encode the digital material into the shortened phonemic representation at the time of input, store it in the encoded form, and then, by means of decoding, reproduce the material in its original digital form. Such a procedure can be properly termed circular recoding because it consists of an encoding-storage-decoding sequence.

The results of phonemic recoding of digital information were more dramatic when phonemic recoding was applied to large amounts of information retained for longer periods of time (Slak, 1971). The effects of phonemic recoding were not limited to the digital form of information. In another study (Slak, 1972), subjects learned a phonemic code for recoding of bivariate figural items (varying in shape and color) into pronounceable two-phoneme syllables (consisting of a consonant followed by a vowel). When subjects used the phonemic code, memory span was significantly higher than when they used natural language code to verbally represent bivariate figures. Since the group mean memory span in the phonemic code condition was compared with the group mean memory span in the natural code condition, it was not possible to ascertain in what subjects the effect was significant or whether the effect generalized over all subjects used.

The purpose of the present study is to verify the effect of phonemic recoding of bivariate figural information on a different type of material and in individual subjects, requiring repeated testing of the same code-trained subjects. It was predicted that the phonemic recoding would increase the subject's memory span for figural items.

\section{METHOD}

\section{Subjects}

One undergraduate female student (Subject 1) and one graduate female student (Subject 2) served as subjects in repeated code-learning and experimental testing sessions. Subject 1 participated on a voluntary basis; the participation of Subject 2 was part of assistantship duties.

\section{Materials}

Lists of figural items drawn on memory drum tape were prepared. Items were figures varying in two dimensions: orientation of line and number of dots. Lines were black and .5 in. long, in one of four different orientations: horizontal, vertical, rising diagonal, and falling diagonal (rising and falling in the reading direction). Each line had zero, one, two, or three dots. In the case of one dot, the dot was placed in the middle of the line. When two dots were used, they were placed at the endpoints of the line. With three dots, two were at the endpoints and one was in the middle. Four different orientations and four different numbers of dots yielded 16 possible figural items. Each list consisted of a certain number of such items in a completely random order.

Twenty random lists of figural items were prepared for each list length from a minimum length of two items to a maximum length of 10 items, or a total of 180 lists. A Stowe memory drum was used for list presentation.

For code-learning purposes only, a large number of random lists of figural items were prepared on regular typing paper. 
For each such list of figural items, an equivalent list of the phonemic version of items (see below) was prepared.

\section{Phonemic Code}

A phonemic code was defined so that each figure could be translated into a pronounceable two-phoneme syllable, consisting of an initial consonant for orientation and a vowel standing for the number of dots. Phonemes for "horizontal," "vertical," "rising diagonal," and "falling diagonal," respectively, were /m/ as in "may," /p/ as in "pay,"/s/ as in "say," and $/ g /$ as in "gay." Phonemes for zero, one, two, and three dots, respectively, were /ee/ as in "see," /ey/ as in "they," /er/ as in "paper," and /ow/ as in "cow." Using this code, a horizontal line with one dot becomes "mey" and a vertical line with three dots, "pow."

\section{Code Learning Procedure}

First, the subject was familiarized with the figural items, and the structure of the phonemic code was explained to her. Then, she was asked to memorize the coding rules. This was followed by 10 hourly code-learning sessions. During the first $8 \mathrm{~h}$ of code learning, the subject practiced the encoding: She read aloud lists of figural items (specially prepared for training purposes) in terms of the phonemic code. The experimenter holding the phonemic version of the list in his hand, corrected the subject each time she made an error. After $8 \mathrm{~h}$ of code learning, the subject was able to read the figural items phonemically at a rate of about $1 \mathrm{item} / \mathrm{sec}$ practically without errors. The last $2 \mathrm{~h}$ of code learning were spent for (1) decoding practice, where the experimenter read items aloud phonemically and the subject drew the corresponding figures; (2) reading items aloud in terms of natural language code (using the words "horizontal," "vertical," "rising," and "falling" to represent the orientation dimension, followed by the words "zero," "one," "two," and "three" to represent the number of dots); and (3) reading the items phonemically as during the first $8 \mathrm{~h}$. At the end of 10 distributed hours of code learning, the subject's performance was judged to be sufficiently good to enable her to participate in the experiment proper.

\section{Testing Procedure}

There were two experimental conditions, designated as the phonemic code condition and the natural language condition. In both conditions, lists of various lengths were presented to the subject on a memory drum at a rate of $1 \mathrm{item} / 2 \mathrm{sec}$. In the phonemic code condition, the subject read items aloud in phonemic code during presentation and, at the time of recall, reproduced the figural items. In the natural language condition, she read the items aloud in natural language and, at the time of recall, reproduced them in figural form.

Twenty tests were used on each subject, 10 tests for each of the two conditions. A test consisted of a single presentation of each list length, starting with the minimum list length of two items in ascending order until the subject was unable to reproduce correctly two lists in a row. The order of conditions was block randomized with 10 tests/block. Different lists were used in different tests, but the same lists in different random orders were used for the two subjects.

\section{Scoring}

Memory span was determined for each test by adding to 1.5 the number of lists correctly reproduced. Ten memory span scores were determined for each condition and each subject.

\section{RESULTS AND DISCUSSION}

Mean memory spans for the natural language and phonemic code conditions with their respective standard deviations for each of the two subjects separately are presented in Table 1.
Table 1

Means and Standard Deviations of Memory Span Scores for the Natural Language and Phonemic Code Conditions and for the Two Subjects

\begin{tabular}{cccccc}
\hline & \multicolumn{2}{c}{ Natural Language } & & \multicolumn{2}{c}{ Phonemic Code } \\
\cline { 2 - 3 } \cline { 5 - 6 } Subject & Mean & SD & & Mean & SD \\
\hline 1 & 4.1 & .667 & & 5.5 & .843 \\
2 & 5.3 & .789 & & 6.6 & 1.370 \\
\hline
\end{tabular}

Since it was predicted that the memory span would be higher in the phonemic code condition, a one-tailed $t$ test was used to test the difference between the two independent means separately for each subject. Memory span in the phonemic recoding condition was significantly higher than in the natural language condition for both subjects [for Subject $1, \mathrm{t}(18)=4.118, \mathrm{p}<.01$; for Subject 2, $t(18)=2.600, p<.01$ ] . (If a two-tailed test or an F test is used, the difference for Subject 2 is significant only at the .05 level.) In Subject 1 , the memory span in the phonemic code condition was increased by a factor of 1.34 if compared with the natural language condition; in Subject 2, the same increment factor was only 1.25 .

Even though phonemic recoding resulted in a significant increase in memory span, if compared with the natural language code, the increase is relatively moderate if compared with the drastic reduction in the length of verbal representation of a given amount of information counted in the number of phonemes. In the phonemic code condition, a figural item, worth 4 bits of information, is represented by two phonemes only; in the natural language, the same information is represented by two words and a much larger number of phonemes. This outcome is consistent with previous findings on the effects of phonemic recoding on the memory span for digital (Slak, 1970) and figural (Slak, 1972) information. Short-term memory capacity is not limited by either a specific number of bits of information or by a specific length of verbal representation. Instead, memory span seems to be influenced or partially determined by both the amount of information, or the informational load, and the length of verbal representation, or the representational load. In phonemic recoding studies, the informational load is measured in bits of information and the representational load in number of phonemes. In the present study, the informational load per item was kept constant while the representational load per item was manipulated, resulting in an increase in memory span that falls short of the relative amount of reduction in representational load.

In an earlier study, Glanzer and Clark (1962) found that materials representable by shorter verbalizations were more easily remembered. However, the results were essentially correlational. It is in a way unfortunate that this interesting study was not followed up by the authors' actual manipulation of verbal representation of material, an approach that would naturally 
require some form of code learning and availability of subjects for longer periods of time.

In addition to its theoretical relevance, phonemic recoding of information is valuable as a practical mnemonic technique. The effectiveness of traditional phonetic mnemonic systems (reviewed by Higbee, 1977, pp. 135-151) varies, and it is difficult to assess how their effectiveness compares with the effect of phonemic recoding. Phonetic systems reviewed by Higbee consist in translating digits into the corresponding consonants; vowels are inserted between consonants to form meaningful words. Such mnemonic systems are usually applied to digital materials. Phonemic recoding system has been applied to both digital and figural materials (Slak, 1970, 1971, 1972), but there is nothing in the nature of the phonemic recoding system that would limit its applicability to these two forms of information. The effectiveness of the traditional phonetic system is based essentially on meaningfulness (though artificial) of the recoded material, while the phonemic recoding system rests on reduction of the representational redundancy (or excess representational load). It also seems logical to expect that the traditional phonetic system would be easier to learn than phonemic recoding, but the traditional system would seem to require a slower presentation of the material than a well learned phonemic code because of the absence of strict one-to-one correspondence rules.

\section{REFERENCES}

Glanzer, M., \& Clark, W. H. Accuracy of perceptual recall: An analysis of organization. Journal of Verbal Learning and Verbal Behavior, 1962, 1, 289-299.

HigBEE, K. L. Your memory: How it works and how to improve it. Englewood Cliffs, N.J: Prentice-Hall, 1977.

SLAK, S. Phonemic recoding of digital information. Journal of Experimental Psychology, 1970, 86, 398-406.

SLAK, S. Long-term retention of random sequential digital information with the aid of phonemic recoding: A case report. Perceptual and Motor Skills, 1971, 33, 455-460.

SLAK, S. Effecto de la recodificación fonemica sobre la amplitud de la memoria inmediata. Revista Latinoamericana de Psicologia, 1972, 4, 23-33.

(Received for publication July 25, 1979.) 